\title{
A Method To Create Stable Lighting And Remove Specular Reflections for Vision Systems
}

\author{
Gilbert Eaton*, Andrew Busch ${ }^{\dagger}$, Rudi Bartels ${ }^{\ddagger}$ and Yongsheng $\mathrm{Gao}^{\S}$ \\ Griffith School of Engineering \\ Griffith University, Nathan Brisbane, Queensland 4122 \\ July 2017
}

Email: *g.eaton@griffith.edu.au, ${ }^{\dagger}$ a.busch@griffith.edu.au, ${ }^{\ddagger}$ r.bartels@griffith.edu.au, ${ }^{\S}$ yongsheng.gao@griffith.edu.au

\begin{abstract}
A lighting system and method has been developed which has shown in testing to allow quality images to be obtained that are free from two particular problems, specular reflections on the subject, and light intensity variation. These problems both diminish the ability to compare objects for attributes such as colour variation, edges, contours, and many other features. The system developed eliminates specular reflection by using the cross-polarisation configuration, and reduced flickering due to fluctuations in the power supply to negligible levels by constructing a high-power DC source capable of providing sufficient 12 Volt power. These two improvements create an environment suitable for taking high-quality, noise free images at high shutter speeds for the purpose of assessing the quality of strawberries moving on a real-time production line.
\end{abstract}

\section{INTRODUCTION}

When using image processing to analyse images or extract features, one problem faced is the inconsistency of images [1]. For example, when using AC lighting and fast shutter speeds, the image intensity will vary with the intensity of the alternating current in the power supply. Although undetectable to the human eye, this type of "flickering" can give rise to a vast difference in image intensity. Another example is that of specular reflection as shown in Figure 1. The bright reflection appears as white "interference" as the information contained in the pixels of this region are lost. The loss of information may include colour, edge contours, region boundaries or hidden defects. This paper is aimed at describing a method of eliminating these problems in order to increase consistency in acquired images.

This image stability is required, particularly in industrial applications when performing defect inspections, or quality control processes. The environment surrounding the subject must be consistent, and free from saturation and specular reflections. Achieving this will then provide more valuable information and comparability of images due to the removal of noise and inconsistency.

The proposed system ensures that objects imaged for computer vision do not contain specular reflections or saturation, and intensity is stable for comparison purposes.

\section{PRoblem Description}

Specular reflections occur when the source of light can be seen on the surface of the object. This can be either a direct reflection from the light source onto the object and then into

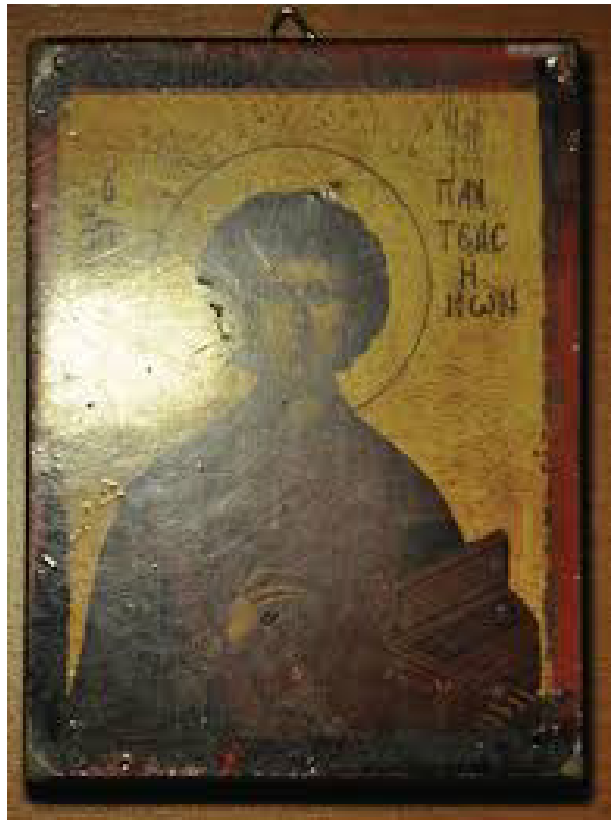

Fig. 1: Example of Specular Reflection on an art display. Note the specular reflection interference with the image causing the loss of information and colour.

the viewer (eye or camera), or through multiple reflections where the light source is still visible. The direction of the specular reflection is related to Snell's Law, in that, the angle of incidence $\left(\theta_{i}\right)$ is equal to the angle of reflection $\left(\theta_{r}\right)$. These reflections are best seen on smooth surfaces such as glass, water, and metal whereas reflections from rough surfaces will result in a more diffuse spread.

According to electromagnetic wave and transmission line theory, at the interface of a good conductor, the reflected field $\left(\bar{E}_{0}^{r}\right.$ - Phasor form of the electric field in the $\mathrm{z}$ plane of incidence) at the interface of the medium is equal to the incident field $\left(\bar{E}_{0}^{i}\right)$ subtract the transmitted field $\left(E_{0}^{t}\right)$ [2] where:

$$
\bar{E}_{0}^{r}=\left(\frac{\eta_{2}-\eta_{1}}{\eta_{2}+\eta_{1}}\right) \bar{E}_{0}^{i}
$$

and for the transmitted field: 


$$
\bar{E}_{0}^{t}=\left(\frac{2 \eta_{2}}{\eta_{2}+\eta_{1}}\right) \bar{E}_{0}^{i}
$$

This shows that the intensity of the reflection is determined by the intensity of the source of the wave and the intrinsic impedance $(\eta)$ of the material and is given by:

$$
\eta=\frac{\omega \mu}{k}=\frac{\omega \mu}{\omega \sqrt{\mu \epsilon}}=\sqrt{\frac{\mu}{\epsilon}}
$$

where the wavenumber $k=\omega \sqrt{\mu \epsilon^{\prime}}$ and $\mu$ is the magnetic permeability of the material. This relates to its susceptibility to magnetic fields, and for diamagnetic and paramagnetic materials (which includes most metals and dielectrics), is considered to be the same as that of free space and $\mu=\mu_{0}=1$ [2].

The electric permittivity, however, is very small for most metals as $\epsilon=\epsilon_{r} \epsilon_{0}=1 \times 8.85 \times 10^{-12}$. Substituting this into equation 3, maintains a large value for intrinsic impedance $\eta_{2}$. thus, the majority of the wave is reflected at this angle. This is comparable to a short circuit on a transmission line resulting in a reflection coefficient of -1 indicating a full reflection even though inverted. This shows that the intensity at the specular angle is not diminished very much by the surface of a smooth conducting surface.

However, one change that can occurs at the interface, is the polarization of the wave. All light sources are randomly generated in all orientations, and can therefore be said to be multi-linear, circularly, or elliptically polarised (commonly named un-polarised or non-polarised) waveform [3], [4].

\section{A. Specular Reflections}

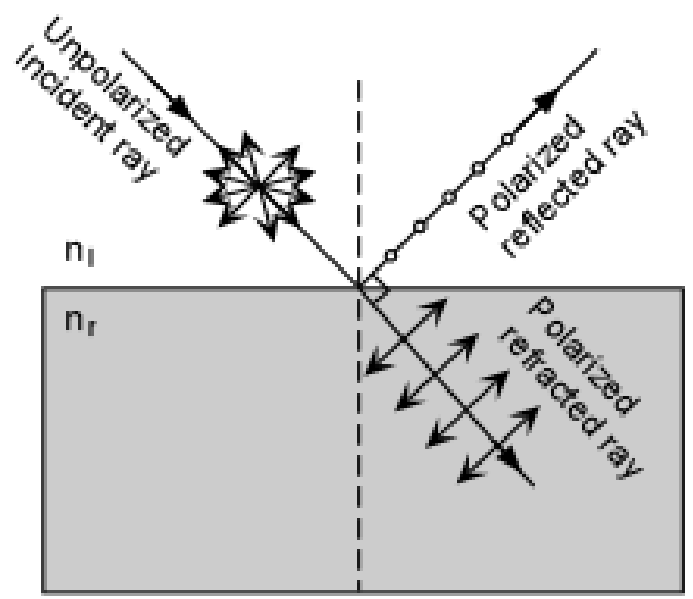

Fig. 2: The interface showing specular reflection of an electromagnetic wave in either the TE or TM orientation.

Figure 2 shows that when an un-polarized incident light ray reflects from a surface, the polarisation is converted to linear form, in the direction of the surface of the object [4].
As the total energy is maintained and the polarisation is converted to linear, the reflection is perceptively intensified in that direction. Multiple reflections can also contribute to this appearance of specular reflection hot spots on objects depending on the surfaces around them and the lighting orientation.

This is a problem for industrial areas where, for instance, on a production line sufficient lighting is required to illuminate the product for image processing purposes. Reflections can occur from the surrounding surfaces or objects, and can be subject to change if the environment surrounding the production line and cameras are rearranged, added or removed.

\section{B. Lighting Intensity Stability}

Alternating Current (AC) power sources have a sinusoidal pattern and have a frequency associated with them. This fluctuation is inherent in everyday power usage, such as the current in mains power supply (in most cases 110/220/240V ( $50 / 60 \mathrm{~Hz}$ ), and is very useful for long distance power transmission. Although this fluctuation can not be seen by the human eye, the fast shutter speeds of the vision cameras can. The difference in the images can theoretically be $100 \%$, as the current passes through the zero point in the sinusoid.

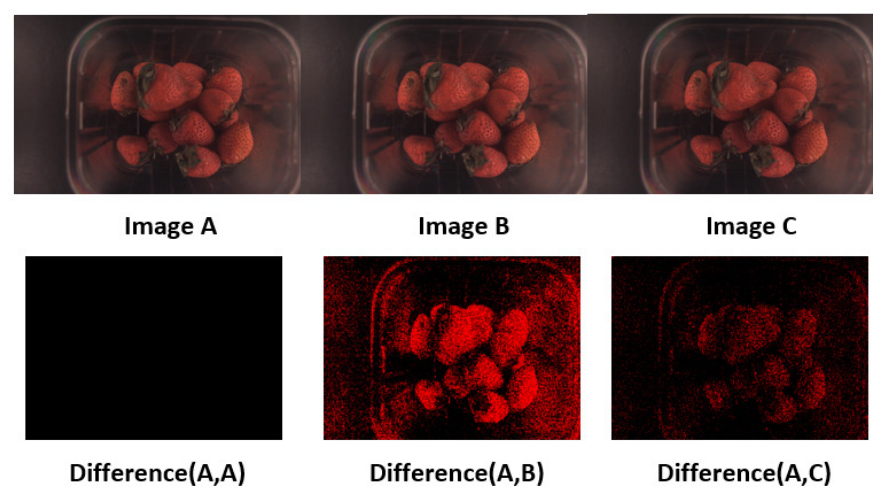

Fig. 3: Differences in pixels between images taken within 1 second. Images 0,2 , and 9 of the random images.

Figure 3 shows the differences between the pixels in three images taken within 1 second using an $\mathrm{AC}$ power source. The images show that over time, using an $\mathrm{AC}$ powered lighting system will create fluctuations in the intensity, potentially causing errors due to the inconsistency of pixel information.

The benefit to using AC lighting is that high power/high intensity lighting is easier to achieve. For example, $1000 \mathrm{~W} 240 \mathrm{~V} 50 \mathrm{~Hz} \mathrm{AC}$ lamps are readily available, however, a $1000 W 12 V D C$ lamp is substantially less common.

This means that to achieve the same level of lighting as an AC system, a $12 \mathrm{~V}$ DC system must be able to power many small LED, tungsten, or halogen lamps resulting in high current requirements. 


\section{ApPLICATION AND RESULTS}

\section{A. System Design}

The acquisition system is comprised of a PC that powers and collects data from the cameras, a light housing that encloses part of the production line, and a power supply. Figure 4 illustrates the topology of the system and the role of each of the main components.

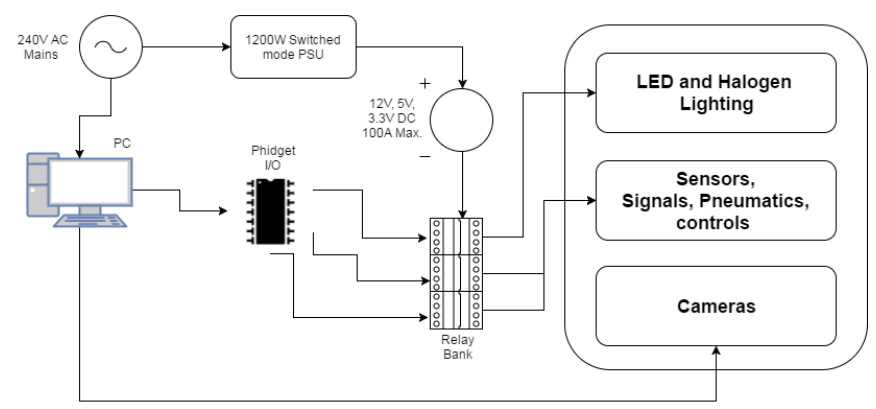

Fig. 4: Overview of the acquisition system's operations.

The PC controls the cameras and peripheral devices such as signals, sensor data, pneumatics, and conveyors through a micro-controller (Phidget). The high-powered devices are connected to a relay bank which is switched by the Phidget and powered by the $1200 \mathrm{~W}$ PSU. The main lighting is turned on only when the system starts to capture images in order to reduce power consumption and heat.

Four cameras are required in order to inspect both the visible and infra-red wavelengths, from above and below. The cameras used are Point Grey Blackfly-BFLY-U3-23S6C-C (Colour), and Blackfly-BFLY-U3-23S6M-C (Mono) that both have a pixel resolution of $1920 \times 1200$, and frame rate of $40 F P S$ using a Sony IMX249 CMOS 1/1.2" sensor. They are fitted with $8 \mathrm{~mm}$, C-mount lenses with a large aperture of $f 1.4$ to allow as much light as possible to reach the sensor.

\section{B. Enclosure Design}

The structure of this system is a lighting enclosure with a production conveyor running through the midpoint for the purpose of capturing strawberry images. Cameras are mounted above and below the v-belt conveyor which supports the strawberry punnet so as to capture images from above and below simultaneously. Using a combination of LED and halogen lamps, the objects pass through the lighting and camera tunnel, imaged, processed, and graded for quality defects.

The enclosure is constructed of laminated white aluminium sheeting curved at the top and bottom in order to disperse the light as much as possible. Diffuse reflections are desired so flat surfaces close to, and in front of, the lighting sources may increase the likelihood of specular reflections. Figure 5 shows the basic skeleton of the enclosure, with the curved top and bottom reflector panels. This skeleton was totally enclosed in the final tests so as to avoid outside interference from ambient AC lighting.

Polarising material has been shown to reduce the effects of these reflections. If surface reflections of the light source

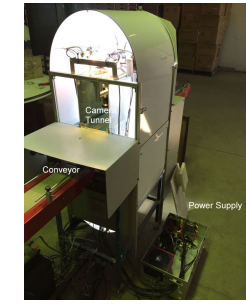

Fig. 5: Lighting enclosure with the conveyor belt running through the centre of the curved top and bottom.

are seen by the cameras, and not the direct light source, the polarising material can remove the specular reflections in one direction. This type of method has been used in experiments to try to improve the quality of images [5], [6].

Exploiting the use of a cross-polarising lens on the camera reduces the specularities even further. By controlling the polarity of the light hitting the objects' surface, and then again before the light enters the camera lens, the reflections in the image are minimised.

If the polarisation orientation is parallel throughout the the inner walls of the tunnel, then all light within the tunnel will have the same orientation of polarization. By adding a polarising filter to the camera lens at an angle orthogonal to the polarised walls, then the specular reflections are dramatically reduced [7], [8].

\section{Power Supply Design}

Preliminary testing gave a good indication of the lighting power requirements by testing many different lighting angles, intensities and polarization configurations. The tests showed that, in order to get adequate intensity through a crosspolariser, the power requirements in Table I are determined to be appropriate:

TABLE I: Power Requirements of the Lighting Devices

\begin{tabular}{cccc}
\hline Type & Camera & Position & Power $(\mathrm{W})$ \\
\hline LED1 & Visible & Above & 72 \\
LED2 & Visible & Above & 72 \\
LED3 & Visible & Above mid & 30 \\
LED4 & Visible & Above mid & 30 \\
LED5 & Visible & Below & 72 \\
LED6 & Visible & Below & 72 \\
LED7 & Visible & Below mid & 30 \\
LED8 & Visible & Below mid & 30 \\
Halogen1 & NIR & Above1 & 35 \\
Halogen2 & NIR & Above2 & 35
\end{tabular}




$\begin{array}{llll}\text { Halogen3 } & \text { NIR } & \text { Above3 } & 35 \\ \text { Halogen4 } & \text { NIR } & \text { Above4 } & 35 \\ \text { Halogen5 } & \text { NIR } & \text { Below1 } & 35 \\ \text { Halogen6 } & \text { NIR } & \text { Below2 } & 35 \\ \text { Halogen7 } & \text { NIR } & \text { Below3 } & 35 \\ \text { Halogen8 } & \text { NIR } & \text { Below4 } & 35\end{array}$

Total $\quad 688$

After initial bench testing, the calculated lighting power was around $688 \mathrm{~W}$, with power draw from peripheral devices totalling approximately $750 \mathrm{~W}$ in normal operating conditions. A $750 \mathrm{~W}$ power supply would meet the operational requirements but leaves no room for variation in current such as inrush, and the potential to increase the amount of light sources in the future. Another requirement of the power supply is that it support $5 \mathrm{~V}$ for peltier, fans, and micro-controller (Phidget), otherwise an additional power supply, or a high-powered, voltage divider circuit will be required.

It is for these reasons that a PC Power Supply Unit (PSU) was determined to best fulfil the requirement. A PSU generally supplies $12 \mathrm{~V}, 5 \mathrm{~V}$, and $3.3 \mathrm{~V}$ as well as some models providing a $-12 \mathrm{~V}$ low current line in order to generate $24 \mathrm{~V}$ potential. These PSUs are designed for longevity, robustness and high power PC applications and hardware used to render video, or facilitate graphics-intensive, high resolution games. The Cougar $1200 W$ PSU is high quality and cost effective means to generate $100 \mathrm{~A} @ 12 \mathrm{~V}$ with the added attractive fault protection features. The image in figure 6 was taken shortly after the power supply was completed and connected to the relevant electronic devices. The relevant specifications are shown in Table II.

TABLE II: Cougar $1200 W$ Power Supply Unit (PSU) specifications

\begin{tabular}{ccccc}
\hline Specification & Min & Typ & Max & Units \\
\hline DC Output & - & - & 1200 & $W$ \\
Operating Temp. & 0 & 25 & 45 & ${ }^{\circ} \mathrm{C}$ \\
Efficiency & 80 & 87 & 90 & $\%$ \\
Transient 12V turn on & 7 & 9 & 10 & $m s$ \\
Ripple 12V (100\% load) & 70 & 73 & 75 & $m V$ \\
Ripple 5V (100\% load) & 39 & 42 & 45 & $m V$ \\
Ripple 3.3V (100\% load) & 40 & 42 & 44 & $m V$ \\
\hline
\end{tabular}

The PSU also has the following protections as part of the manufacturing specifications:

- Over Voltage Protection - If the voltages increase above a certain tolerance value on the single lines, the PSU automatically switches off.

- Under Voltage Protection - If the voltages fall below a certain tolerance value on the single lines, the PSU automatically switches off.

- Over Power Protection - If the system is oversized and requires more power from the PSU than it can deliver, this protection function is activated.

- Over Current Protection - If the load on a single line is higher than indicated, the PSU automatically switches off.

- Short Circuit Protection - In the case of a short-circuit this feature prevents damage to the core components of the PSU and its system components.

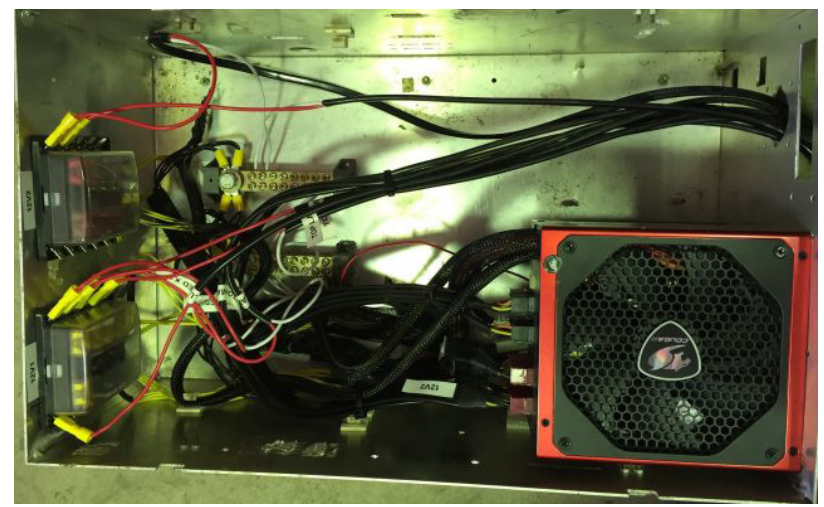

Fig. 6: Power Supply box with PSU mounted, fuses, ground terminals, and wiring to the electronics through upper right wall.

\section{Results}

The $1200 W$ DC power supply is designed and constructed so that the full power of the $12 \mathrm{~V}$ rails are supplied by distributing each of the two channels between the top and bottom lighting circuits. The power supply also supplies power to peripheral devices such as pneumatics, LED indicators, warning lights, logic control board, sensors, peltier, and fans.

The resulting images taken using the designed enclosure have dramatically improved the image stabilisation when compared to the AC lighting system. The DC-powered lighting system resulted in very small intensity fluctuations, and was measured to be $<0.1 \%$ as seen in Figure $7 \mathrm{a}$ and $7 \mathrm{~b}$. The average pixel area for each image was obtained by first taking a threshold of subjects in the each of the frames:

$$
R=\sum_{i=1}^{P} t_{1} \leq x \leq t_{2}
$$

with $P$ denoting the number of pixels in each image and $t_{1}, t_{2}$ the threshold limits. The average region area $\left(A r e a_{\text {avg }}\right)$ was calculated and is given by the mean definition:

$$
\text { Area }_{\text {avg }}=\bar{R}=\frac{1}{N} \sum_{i=1}^{N} R_{i}
$$


where $N$ is the number of images to average, and $R_{i}$ represents the number of region pixels in each image.

Data was gathered twice, once for a period of two seconds with a fast frame rate, and the other over a three hour period in order to assess the case of high speed cameras, and in the case of constant operation for extended periods of time.

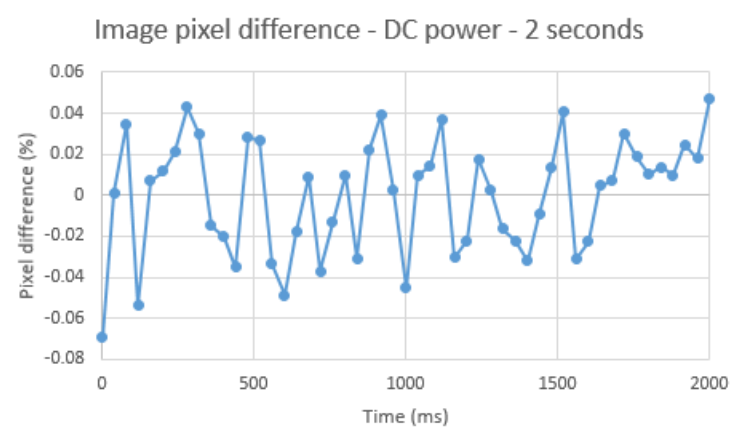

(a)

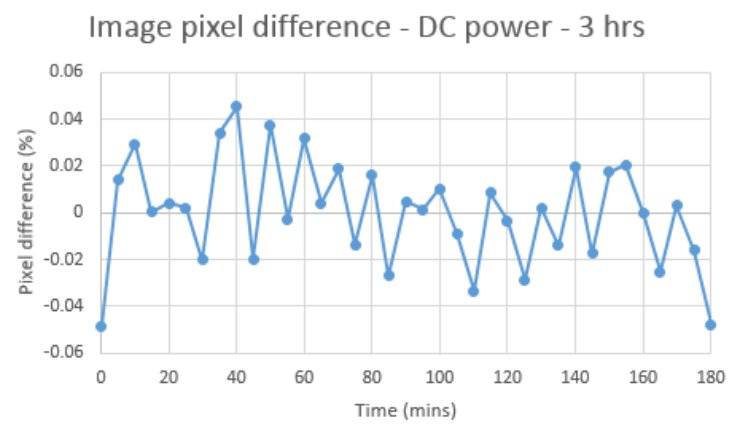

(b)

Fig. 7: Intensity measurements taken using DC lighting system showing the small variations over: a) A two second period and b) A 3 hour period. All results have a difference of less than $0.1 \%$.

In order to solve the specular reflection problem, the use of a cross-polarization lighting design was implemented. As discussed in Section III-B, polarizing material is installed vertically (polarization direction can be either orientation, although further testing may indicate a preference) to form a tunnel through the enclosure. The second polarizer is an adjustable filter fitted to the lens of the visible camera. The graph shown in Figure 8 shows that when the specular pixels are removed, the region pixels are increased giving a better assessment of the area of interest.

Figure 9a shows an image with only one polarizer in place allowing some specular reflections to be seen (the amount of specularities would be even greater if the acquisition had no polarizing elements), then by rotating the camera lens polarizer, images were taken at 16 positions from $0^{\circ}$ to $180^{\circ}$.

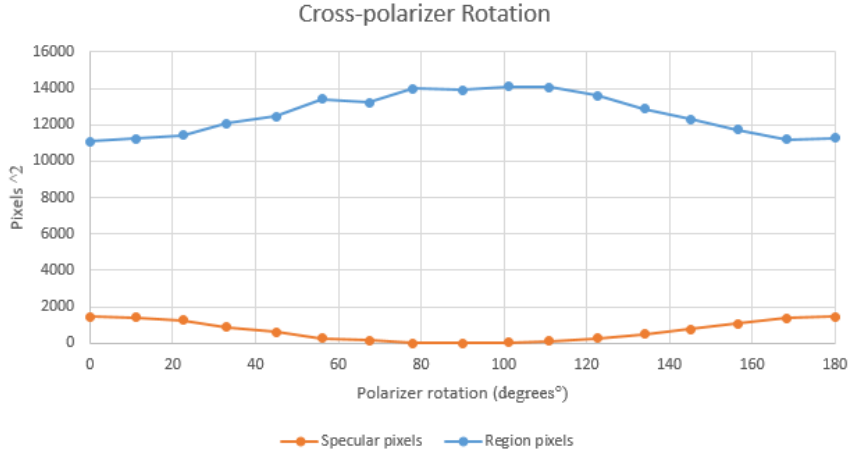

Fig. 8: Experimental results gathering the specular and region pixels, whilst rotating the cross-polariser on the camera lens.

When the polarizers are orthogonal $\left(90^{\circ}\right)$, the result is a more flattened image that removes the specular reflections almost entirely. Figure $9 \mathrm{~b}$ depicts this change and it can be seen that the surface of the strawberries are much clearer and will allow better image processing due to the increase of pixels which can be analysed, and a decrease in noise.

\section{CONCLUSION}

Image acquisition plays an important part in any machine vision application to ensure that the images acquired are free of distortion and provide the most information. Lighting is therefore an important factor in image acquisition systems, allowing objects surfaces to be illuminated with as little interference as possible.

A system has been developed to inspect strawberries and assess them for quality attributes. The first step in the development is to design and construct the acquisition system and integrate it with the production line. After the enclosure had been installed, the LED/halogen lighting, polarizers, and cameras were mounted. The DC power supply delivers the required amount of power at a constant current which ensures that the intensity fluctuations between images are insignificant. Experimental results show that the fluctuations are less than $0.1 \%$ of total intensity, even when operating for many hours. Experiments were also performed that showed that the crosspolarization technique has the ability to entirely remove of the specular reflections in most cases, whilst providing more pixels that can be analysed in each frame.

The system developed is extremely successful in achieving the desired consistent image acquisition. The system can image 2 punnets of strawberries per second on the production line and the image quality is illuminated, free from interference, and consistent between images, even with a shutter speed as fast as $1 \mathrm{~ms}$. Future work includes the strategy to strobe the lighting in order to reduce power usage and heat, as well as the introduction of image processing algorithms to perform the assessment. 


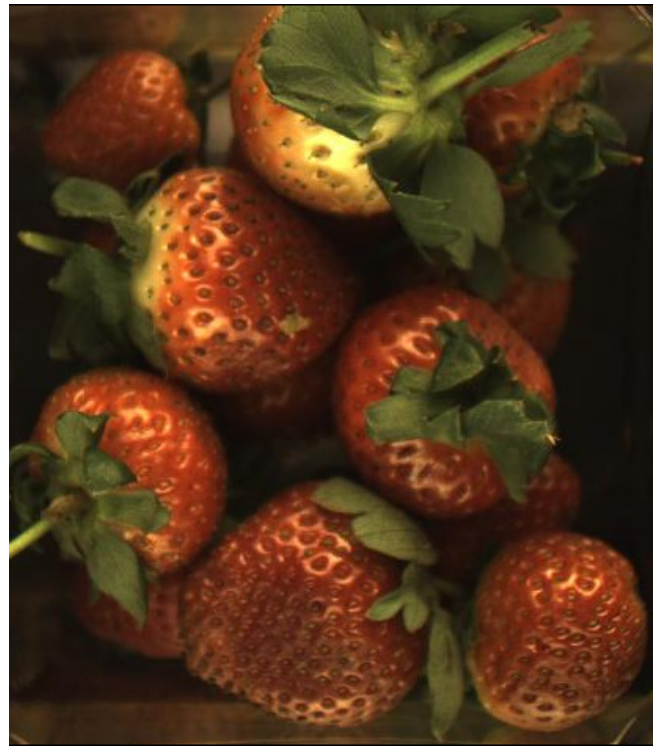

(a)

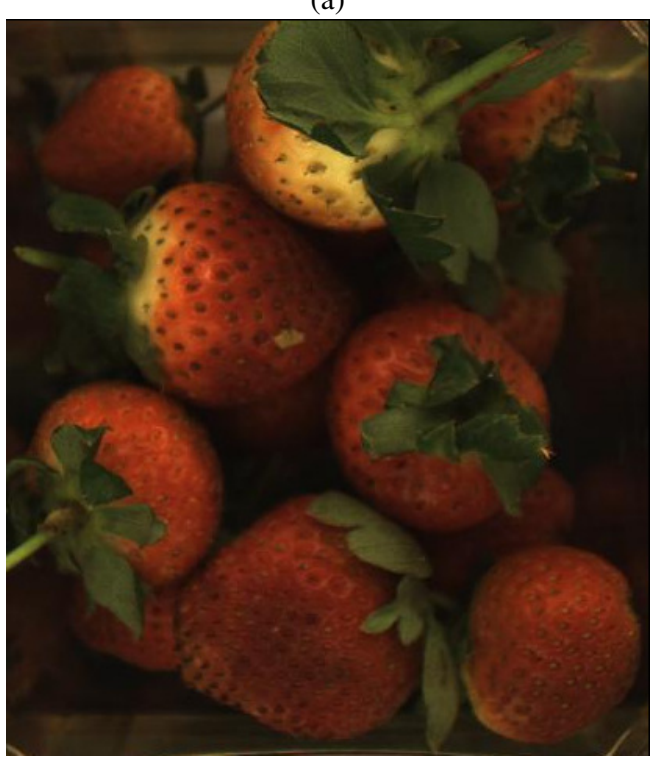

(b)

Fig. 9: Sample images of strawberries with polarizer in position: a) $0^{\circ}$ and b) $90^{\circ}$. These images illustrate the loss of information in a strawberry image where glossy pixels create noise.

\section{ACKNOWLEDGMENT}

The authors would like to acknowledge the ARC linkage program as well as Magnificent Pty. Ltd for their ongoing support of this project.

\section{REFERENCES}

[1] A. A. et al, "A survey of specularity removal methods," COMPUTER GRAPHICS forum, vol. 30 (2011), number 8.

[2] F. T. Ulaby and U. Ravaioli, Fundamentals of Applied Electromagnetics. Global: Pearson, 2015.

[3] A. A. et al, "A survey of specularity removal methods," Journal of Chemical Physics, vol. 10.
[4] P. S. A. Nelson, "Properties of light and examination of isotropic substances," 2014

[5] G. A. A. et al, "Recovery of surface orientation from diffuse polarization," IEEE TRANSACTIONS ON IMAGE PROCESSING, vol. 1057-7149.

[6] L. B.Wolff and T. E. Boult, "Constraining object features using a polarization reflectance model,," IEEE Trans. Pattern Anal. Mach. Intell, vol. 13, no.7.

[7] R. Anderson, "Polarized light examination and photography of the skin,," Arch Dermatol, vol. 127.

[8] R. V. K. et al, "Complementary use of cross-polarization and standard oct for differential diagnosis of pathological tissues," OPTICS EXPRESS, vol. Vol: 10, no.15. 